\title{
Work ability assessment among acutely admitted patients using biomarkers
}

\author{
Ove Andersen", Linda Camilla Andresen ${ }^{1}$, Louise Lawson-Smith', Lea Sell ${ }^{2}$ and Inge Lissau ${ }^{1 *}$ (D)
}

\author{
* Correspondence: \\ info@ingelissau.dk \\ ${ }^{1}$ Clinical Research Centre, \\ Copenhagen University Hospital, \\ Hvidovre Kettegård Alle 30, 2650 \\ Hvidovre, Denmark \\ Full list of author information is \\ available at the end of the article
}

\begin{abstract}
Background: Can biomarkers be used to predict the future work ability of patients admitted to hospital? To answer this question, we use a combination of biological measurements, registry data, data from questionnaires, and results from functional tests. The study comprises both a randomized controlled intervention trial with physical training and a prospective study, the latter using only questionnaire data combined with registry data. The setting is an emergency department at a hospital in the capital region in Denmark. Participants to the study were recruited right after admittance to the hospital.

Here we present the study background and method as well as the initial recruitment of project participants.
\end{abstract}

Results: Patients eligible for the intervention trial were randomized in blocks to either the intervention or the control group. The randomization was stratified first according to age-group and second by whether the diagnosis was mainly physical or mental in origin. Data was collected in four rounds: at admission to hospital, at the intervention site 2 weeks after discharge, 12 weeks after discharge when the training program was completed and via a forwarded questionnaire on work ability after 12 months.

In the prospective study, the participants answer questionnaires on work ability and physical and mental resources at admission to hospital, and will be evaluated in regard to work ability, labour market participation and sick-leave after 2 years. Work ability is measured using a validated questionnaire, the Work ability Index (WAI), and data on labour market position and sick-leave are gained from national registries.

Conclusions: The strength of the study is that it includes biomarkers, results from questionnaires and functional tests along with socio-economic background and registry data. Thus, it reflects the complexity of work ability.

The short duration of stay in the Emergency Department and the high level of activity here made it difficult to have enough time with the patients to fill in the questionnaires and provide information on the project. Therefore, it should be considered if another more appropriate time point for these activities can be examined.

The study presents new opportunities for big data analysis within the health care sector which may be especially adaptable within a Danish context since health registries are already of high quality here.

Trial registration: ClinicalTrials.gov at October 9, 2015 identifier; NCT02614430. Retrospectively registered.

Keywords: Physical activity, Training, Sick-leave, suPAR, Biomarkers, Inflammation, Work ability, Intervention, Rehabilitation, Hospitalization

\section{Biomed Central}

(c) The Author(s). 2017 Open Access This article is distributed under the terms of the Creative Commons Attribution 4.0 International License (http://creativecommons.org/licenses/by/4.0/), which permits unrestricted use, distribution, and reproduction in any medium, provided you give appropriate credit to the original author(s) and the source, provide a link to the Creative Commons license, and indicate if changes were made. The Creative Commons Public Domain Dedication waiver (http://creativecommons.org/ publicdomain/zero/1.0/) applies to the data made available in this article, unless otherwise stated. 


\section{Background}

Facing an ageing work force, prevention of premature exit from the labour market is more important than ever. Earlier studies have shown that reductions in work ability from the age of 50 years raise the risk of future disability pension, $[1,2]$ and maintaining the work ability among vulnerable groups should be prioritized.

Work ability is influenced by many different parameters such as health, functional capacities, education, competencies, values, attitudes and degree of motivation along with the actual job situation [3]. In this study, we focus on the relationship between health and work ability using a combination of biological measurements, registry data, along with results from questionnaires and functional tests to assess the progress in work ability. As a biological measure of health and the development in health status, we use the biomarker suPAR indicating the degree of low grade inflammation.

In earlier studies, we have shown that suPAR is a highly stable inflammatory biomarker associated with disease progression and severity. Elevated suPAR levels have been observed in a wide range of pathological conditions, including both infectious diseases and non-communicable chronic diseases, such as diabetes, cardiovascular disease, and cancer. Also, SuPAR levels are associated with lifestyle factors, such as smoking, alcohol intake, and sedentary lifestyle [4]. By applying a cluster of biomarkers, such as interleukin (IL)10, IL6, tumour necrosis factor (TNF)-alfa, and suPAR, the cellular and functional aspects of aging can be comprehended across diseases. This is feasible to execute and implement in the acute care setting, and can be used to assess biological aging and general health status.

Health and functional capacity can be regarded as the foundation of work ability $[5,6]$. Poor health and chronic illness have been found to significantly increase the risk of exit from paid employment and disability pension [7]. Moreover, poor health behaviour in regards to life style factors, such as smoking and overweight, have been linked to reductions in work ability $[8,9]$. Results from cross-sectional analyses of the effects of work-related and individual factors on work ability show that low work ability is also associated with a lack of physical activity, poor musculoskeletal capacity, and physical conditions [10].

After the age of 50, it may become critical for the individual to stay on the labour market, especially if the working conditions are physically strenuous [3]. If biomarkers prove to be effective in predicting future work ability, it is possible to identify patients at risk of later reduced work ability and early preventive measures are therefore possible. The choice of utilizing acute admission as a fixed point for assessing work ability is supported by studies showing that admission to hospital imposes a risk of functional decline [11, 12].

Physical training has been demonstrated to prevent early decline in work ability [13]. In addition, several work site intervention studies involving physical training have reported an improved physical capacity and prevention of deterioration of work ability among the employees [14]. In the study presented here, the effect of physical training on recovery and work ability is investigated, using the results from biomarkers and functional test as indicators of recovery. 


\section{Methods}

\section{Aims and research questions}

The project consists of two parts: an intervention trial and a prospective study. The aim of the intervention trial is to evaluate the effect of an early physical training intervention on inflammatory indicators, physical and mental functioning, and health-related quality of life, among patients who were acutely admitted previously. This should provide answers to the following research questions: Can physical training after discharge from acute hospitalization reduce the inflammatory level in the blood? Can physical training after discharge from acute hospitalization improve physical and mental functioning? Can biomarkers, either alone or combined with questionnaires and functional tests, be used to predict work ability and labour market status a year after the admission to a hospital?

The aim of the prospective study is to investigate if it is possible by means of questionnaires on work ability to identify patients at risk of future reduced work ability during an acute hospital stay. More specifically, the research question is whether self-reported work ability and expectations to future work ability during acute hospital admission can predict work ability, labour market participation and number of sick days after 2 years.

\section{Setting}

The Emergency Department at Amager Hvidovre Hospital consists of two geographically separated departments with an overall intake of approximately 150-175 patients per day. The patients have a mean age of 67 years (range 15-104 years). The emergency room is a part of the public health care system, which provides feeless, tax-paid primary and secondary medical care. The Emergency Department offers care for patients with acute illnesses and determines if they require further access to specialized health care. This function of triage makes the Emergency Department a place of high activity [15].

A previous study in the same department pointed to that the maintenance of a continuous patient flow generates an organizational culture in which actions that secure available beds are collectively prioritized by the practitioners, referred to as a flow culture [16]. This means that actions that the clinical personnel perform in order to allocate patients, such as the nurses measuring vital parameters, are prioritized ahead of other routines [17].

\section{Study population}

The population base for this trial is medical patients acutely hospitalized in the Emergency Department at University Hospital of Amager Hvidovre, the Capital Region of Denmark. An acute hospitalization is here defined as an unplanned personal contact to a hospital due to health-related problems.

Patients were eligible for the study if aged 18 to 60 years and expected to be hospitalized for at least $12 \mathrm{~h}$. All participants should also be entitled to time-limited sickness or unemployment benefits from either the employer or the municipality.

In addition, the participants for the intervention trial should have an increased risk of a loss of work ability (earning capacity). The assessed risk is based on known risk factors for loss of work ability: self-reported risk of decreased work ability after 
discharge, presence of one or more chronic or long-term diseases, or pain in the joints and muscles within the week before admittance. The patient was included in the study if fulfilling one or more of these three criteria. Exclusion criteria are inability to cooperate, having an end-stage disease, or major trauma.

\section{Recruitment of patients}

The participants for both studies were recruited in the emergency department by a project nurse or project assistant. The participants for the prospective study were asked to fill in a questionnaire during their current hospitalization. The patients who met the inclusion criteria for the intervention trial were given additional written information on the project. The same or the following day these patients were contacted again and if he or she agreed to participate, a declaration of consent was signed, and they had to answer a questionnaire regarding his or her daily functioning. Further, the project nurse supervised the participant as they performed gait speed and hand-grip strength tests, and collected a blood sample. Finally, the participant was informed of what should happen at the next visits.

Two weeks later, participants for the intervention trial meet with both the physiotherapist and the project nurse, and answer the questionnaires and perform hand-grip strength and habitual gait speed tests again. The project nurse collects another blood sample. The participant is then interviewed by the physiotherapist regarding training potential and the need for training. Based on these answers, it is decided if the patient is suited to participate in training sessions and if so, he or she is randomized to either the intervention with training or the control group with no training.

After the randomization, the participants in the control group make an appointment for the last visit, whereas the intervention participants receive additional information from the physiotherapist.

\section{The physical training program}

The physiotherapist develops individual training plans for all the participants in the intervention group, covering both basic and work-related needs. Training takes place two to three times a week for $1-2 \mathrm{~h}$ at a time, in 4 to 9 weeks. When the training is completed, the physiotherapist writes an evaluation, including attendance to the training sessions and work status when completing the training sessions.

After 12 weeks, the whole of the intervention group has completed the training and both the intervention and control groups are invited for a last visit to the hospital for blood tests, followed by hand-grip strength, habitual gait speed tests, and questionnaires.

\section{Data collection in the intervention trial}

The participants in the intervention trial will be evaluated in regard to self-reported work ability, health related quality of life and sick-leave, physical and mental functioning, and inflammatory level in the blood (biomarkers). Data will be collected in four rounds: at admission to the hospital or just after, 2 weeks after discharge (before onset of the physical training programme), 12 weeks after discharge (when the physical training is complete), and by a questionnaire on work ability, after 12 months. See Table 1 for a full description of tests and measures.

\section{Data collection in the prospective study}

The participants for this study will be evaluated using questionnaires on admission to the hospital, and at a follow-up after 2 years. This group will be evaluated with regard 
Table 1 Measurements in the intervention trial

\begin{tabular}{|c|c|c|c|c|}
\hline & $\begin{array}{l}\text { Time of } \\
\text { admission }\end{array}$ & $\begin{array}{l}\text { Two weeks } \\
\text { after admission }\end{array}$ & $\begin{array}{l}\text { Twelve } \\
\text { weeks }\end{array}$ & $\begin{array}{l}\text { One } \\
\text { year }\end{array}$ \\
\hline Self-reported work ability (WAI) & $x$ & $x$ & $x$ & $x$ \\
\hline Gait speed & $x$ & $x$ & $x$ & \\
\hline Hand-grip strength & $x$ & $x$ & $x$ & \\
\hline Health-related quality of life (EQ-5D) & $x$ & $x$ & $x$ & $x$ \\
\hline $\begin{array}{l}\text { Blood tests (suPAR, INR, DIFFMAS, HB, MCHC, MCV, } \\
\text { THROM, ALAT, ALB, BASP, BILI, CARB, CREA, CRP, } \\
\text { eGFR, K, LDH, NA) }\end{array}$ & $x$ & $x$ & $x$ & \\
\hline Major Depression Inventory (ICD-10) & $x$ & $x$ & $x$ & \\
\hline Self-reported sick-leave & & & & $x$ \\
\hline
\end{tabular}

to work ability, labour market participation and absence from work due to illness, after 2 years. See Table 2 for a full description of measures.

\section{Measures}

Blood tests

From the blood samples, inflammatory receptors, cytokines and chemokines are analyzed. The biomarker suPAR indicates the degree of low grade inflammation. SuPAR is the soluble form of the urokinase-type plasminogen activator receptor (UPAR). UPAR is involved in plasminogen activation [4], along with other cytokines involved in the inflammatory response. The cluster of biomarkers IL10, IL6, TNF-alfa, and suPAR reflect the cellular and functional aspects of aging across diseases. The analyses will be carried out as batch after sample collection using ELISA and/or Luminex technology.

\section{Work ability}

The questionnaire items used to assess the work ability of the participants were primary from the Work Ability Index, WAI, a well-validated questionnaire used for health examinations and workplace surveys [18].

As the WAI is a comprehensive questionnaire, it is not entirely suited for the flow of patients through an emergency department. Consequently, we used only some of the items from the WAI at the baseline. Other studies have used single items as a measure of work ability with good results [8, 19-21]. For the follow up-studies, the whole of WAI is used.

Another consideration when evaluating work ability is that because the questionnaire is here answered by a person in a non-stabile setting in regards to health, and even while a treatment plan is still being prepared, both the before-admission work ability and the current work ability should be evaluated.

Table 2 Measurements in the prediction study

\begin{tabular}{lll}
\hline & Time of admission & Two years \\
\hline Self-reported work ability (WAl) & $\mathrm{X}$ & $\mathrm{X}$ \\
Incident and/or length of long-term sick-leave & & $\mathrm{X}$ \\
Transition to early retirement pension & $\mathrm{X}$ \\
Voluntary retirement pension & $\mathrm{X}$ \\
\hline
\end{tabular}




\section{Sick-leave}

In the prospective study it is tested if self-reported work ability can predict the outcomes long term sick-leave, transition to early retirement pension, or voluntary retirement pension. These data will be obtained from a national registry on social transfer payments.

Day of recovery is defined as the first day back at work after sick-leave.

\section{Functional tests}

Hand-grip strength: The development of isometric hand-grip strength is measured for the dominant hand using a handheld dynamometer (Digi-II; Saehan).

Habitual gait speed: The development in degree of disability is estimated using a walking test $(6 \mathrm{~m})$. The participants are instructed to walk at their habituated pace on a marked 8-m field. An average is calculated after two rounds.

\section{Mental resources}

Mental functioning is evaluated using the Major Depression Inventory (MDI). MDI is a questionnaire comprising 10 symptoms that are parts of the World Health Organization's definition of depression [22].

Health

Health-Related Quality of Life is measured using the EQ-5D: The EQ-5D is a validated questionnaire measuring health status. The questionnaire provides a simple descriptive profile and a single index value.

\section{Other health data}

Readmission to hospital: Information on any readmissions from the day of discharge to the end of the study period will be obtained from the National Patients Registry.

Medication: Data on medication use will be subtracted from a national registry on prescriptions kept by Statistics Denmark. This can be used as outcome control variable in the statistical analysis.

Doctor visits: Data on visits to the doctor will be subtracted from the registry of the National Health Insurance kept by Statistics Denmark. This can be used as outcome control variable in the statistical analysis.

Medical diagnoses: Data on patient diagnoses will be obtained from the National Patients Registry. This will be used to measure the severity of disease.

\section{Statistical analysis}

Patients eligible for the intervention study were randomized in blocks to either the intervention or the control group using the statistical programme $\mathrm{R}$. The randomization was stratified first according to age-group and second by whether the diagnosis was mainly physical or mental in origin.

In order to calculate the predictive value of the physical tests and biomarkers, we expect to use receiver operating characteristics (ROC) curves and to calculate the total area under the curve. The Youden index [23, 24] will be used to estimate the optimal cut-off point for each predictor and marker. In order to estimate the overall information from the clinical tests in a single prediction algorithm, we will use the method proposed by Xiong et al. [25]. 


\section{Results}

Only a minority of the patients in the Emergency Department were eligible for the study according to the basic inclusion criteria. Applying the additional criterion of being in risk of work ability loss, out of more than 2.811 patients assessed during the total recruitment period of 19 months, only a maximum of 50 patients could be allocated to the training intervention. These patients also had to meet the criteria in regard to ability to participate in the training programme. For the prospective study, 200 patients met the basic study criteria.

The recruitment of patients was difficult for several reasons. Apart from only a minority of patients meeting the basic inclusion criteria, the focus on a constant flow of patients and the high level of activity in the emergency department implied that it was difficult to get in contact with the patients before they have left the emergency room again.

\section{Discussion}

The strength of this study is that it includes biological measures along with functional tests and questionnaires as well as socio-economic background to assess a person's risk of reduced work ability. Thus it reflects the complexity of work ability. By combining these different types of data, the study incorporates and uses a more holistic approach to the patient that goes beyond the time point of data collection at the hospital.

The project presented here also represents new types of collaborations across sectors that involve municipalities and private agents working to promote employment. They are here presented here with an earlier entry point for their preventive measures. The advantage is the possibility of targeting the efforts at a person's current work position instead of the potential unemployment following a decrease in work ability.

The effect of supervised physical training, as used in the intervention trial, is well documented, which adds to the robustness of the study. However, a weakness of the study is that we use both a new measure for predicting work ability and introduce a new type of participant at the training courses, making the intervention more complex.

The project faced five major obstacles in the recruitment of patients: the short duration of stay in the Emergency Department made it difficult to see the patient, the patients were stressed due to the acute spell of serious illness, the surroundings at the Emergency Department did not allow for private conversations about the project with the patient in a separate room, the treatment plan had not yet been outlined when the patient was asked about their work ability, and in regard to participating in the intervention trial, the training only was offered in the daytime. The reason for the last challenge is that the training concept usually involved individuals on sick-leave or who had lost their current employment, whereas the intervention participants mostly work during the daytime.

\section{Conclusion}

The project will provide new knowledge on inflammatory biomarkers and their ability to predict the future work ability of patients. Since this is a tool which is both easy and economically to apply in a health care context, it has the potential to facilitate new settings for the prevention of reduced work ability. It adds to the strength of the study, 
that it not only includes biomarkers, but also results from questionnaires and functional tests in combination with registry data.

The short duration of stay in the Emergency Department and the high level of activity here made it difficult to have enough time with the patients to fill in the questionnaires and provide information on the project. Therefore, it should be considered if another more appropriate time point for these activities can be examined if similar projects are planned.

With its unique combination of data, the study also presents new opportunities for big data analysis within the health care sector which may be especially adaptable within a Danish context since health registries are already of high quality here.

\section{Trial status}

Patient recruitment has been completed. Data collection will continue with 12-month and 2-year follow-ups.

\section{Abbreviations \\ EQ-5D: Health-related quality of life; MDI: Major depression inventory; suPAR: The soluble form of the urokinase-type Plasminogen Activator Receptor (UPAR); WAI: Work ability index}

Acknowledgements

The authors want to thank Steen Ladelund and Line Jee Hartmann Rasmussen for providing statistical support.

\section{Funding}

This study was founded by Markedsmodningsfonden (grant no. 2014-8257).

\section{Availability of data and materials}

Not applicable.

\section{Author contributions}

OA and LCA conceived the study, are project leaders, wrote the study grant, and are grant holders. LLS entered the study in Clinical Trials. IL drafted the first version of the manuscript. IL, LS, LCA, OA, and LLS revised the paper. LS contributed to the methods on work ability measurements. All authors contributed to refining the study and approved the final manuscript.

\section{Ethical approval and consent to participate}

The study has been approved by the Regional Ethical Committee (registration number.

H-15005411). In Danish: De Videnskabsetiske Komiteer for Region Hovedstaden, Kongens Vænge 2, 3400 Hillerød

Phone +45 386663 95, mail: vek@regionh.dk;

webpage: regionh.dk/vek.

The studies are being carried out in accordance with the principles of the Declaration of Helsinki. Participants are covered by the Clinical Research Centre, Amager and Hvidovre Hospital's Patient Insurance through the Capital Region of Denmark. The collected data will be kept in accordance with the Data Protection Agency guidelines.

The project manager works with the clinical staff from the participating departments responsible for contacts with all patients who meet the inclusion criteria for the studies. Patients who met the inclusion criteria received written and verbal information about the project, including the advantages, disadvantages, risks, and patient rights of participating in the project. The patients were contacted by the project manager the day after the verbal and written information was received. If the patient wished to participate in the project, the project manager obtained the consent form signed by the participant, and scheduled a time for tests and blood sampling.

\section{Consent for publication}

The manuscript does not contain any personal data. The data collection is still ongoing, and they are at present stored according to Danish data legislation on the area. The type of data does not require public availability, and since we're planning a number of original papers using the data, is also not appropriate to deposit the data in publicly available repositories. All data from Danish Health Care projects independent of project type, data collected, means of compilation and/or analysis which is based on Danish medical patients, samples, records or derivate must comply with the Danish Data Protection Agency in concordance with current guidelines (The Act on Processing of Personal Data, Act No. 429 of 31 May 2000), motion 528 (Sikkerhedsbekendtgørelsen) stipulating that access requires a 3rd Party Data-analysis agreement with the Capital Region of Denmark in order to receive semi or fully anonymized data for placement or repository in public domains. All users of said data must comply with the above.

\section{Competing interests}

$\mathrm{OA}$ is inventor of the patent on suPAR and risk. Hvidovre Hospital, Denmark, owns the patent which is licensed to ViroGates A/S. All other authors declare that they have no competing interests. 


\section{Publisher's Note}

Springer Nature remains neutral with regard to jurisdictional claims in published maps and institutional affiliations.

\section{Author details}

${ }^{1}$ Clinical Research Centre, Copenhagen University Hospital, Hvidovre Kettegård Alle 30, 2650 Hvidovre, Denmark. ${ }^{2}$ Department of Economy, Copenhagen University Hospital Bispebjerg - Frederiksberg Hospital, Bispebjerg Bakke 23 Entrance 20 C, 2nd floor, 2400 Copenhagen, Denmark.

Received: 15 November 2017 Accepted: 6 December 2017

Published online: 20 December 2017

\section{References}

1. Liira J, Matikainen E, Leino-Arjas P: Work ability of middle aged Finnish construction workers - aa follow-up study in 1991-1995 2000, 25:477-481.

2. Roelen CAM, Heymans MW, Twisk JWR, JJLvd K, Groothoff JW, WV R. Work ability index as tool to identify Workers at Risk of premature work exit. J Occup Rehabil. 2014;24(4):747-54.

3. IImarinen JE. Aging Workers. Occup Environ Med. 2001;58(8):546-546.

4. Kofoed K, Schneider UV, Scheel T, Andersen O, Eugen-Olsen J. Development and validation of a multiplex add-on assay for sepsis biomarkers using XMAP technology. Clin Chem. 2006;52(7):1284-93.

5. IImarinen J. Towards a longer and better working life: a challange of work ageing. Med Lav. 2006;97:5.

6. IImarinen J. Work ability—a comprehensive concept for occupational health research and. Prevention. 2009;35(1):1-5.

7. Rijn RMv, Robroek SJW, Brouwer S, Burdorf A. Influence of poor health on exit from paid employment: a systematic review. Occup Environ Med. 2014;71(4):295-301.

8. Sell L, Faber A, Søgaard K. Impacts from occupational risk factors on self reported reduced work ability among Danish wage earners. In: Promot Work Product. Hanoi Vietnam: Taylor and Francis; 2007. p. 105-12.

9. Hasselhorn HM, Tackenberg P, Müller BH: Working conditions and intent to leave the profession among staff in Europe. In. University of Wuppertal; 2003.

10. Berg TIJvd, Elders LaM, Zwart BCHd, Burdorf A. The effects of work-related and individual factors on the Work Ability Index: a systematic review. Occup Environ Med. 2009;66(4):211-220.

11. Sager MA, Franke T, Inouye SK, Landefeld CS, Morgan TM, Rudberg MA, Sebens H, Winograd CH. Functional outcomes of acute medical illness and hospitalization in older persons. Arch Intern Med. 1996;156(6):645-52.

12. Boyd CM, Xue QL, Guralnik JM, Fried LP. Hospitalization and development of dependence in activities of daily living in a cohort of disabled older women: the Women's health and aging study I. J Gerontol Series A, Biological Sci Med Sci. 2005;60(7):888-93.

13. Pohjonen T, Ranta R. Effects of worksite physical exercise intervention on physical fitness, perceived health status, and work ability among home care workers: five-year follow-up. Prev Med. 2001;32(6):465-75.

14. Jørgensen MB, Ektor-Andersen J, Sjøgaard G, Holtermann A, Søgaard K. A randomised controlled trial among cleaners-effects on strength, balance and kinesiophobia. BMC Public Health. 2011;11(1):776.

15. Duffield CM, Conlon L, Kelly M, Catling-Paull C, Stasa H. The emergency department nursing workforce: local solutions for local issues. International emergency nursing. 2010;18(4):181-7.

16. Wassar Kirk J, Nielsen P. The influence of flow culture on nurse use of research in emergency care: An ethnoraphic study. Klinisk Sygepleje. 2015;29(2):16-35

17. Kirk JW, Nilsen P. Implementing evidence-based practices in an emergency department: contradictions exposed when prioritising a flow culture. J Clin Nurs. 2016;25(3-4):555-65.

18. Tuomi K, Ilmarinen J, Jahkola A, Katajarinne L, Tulkki A. Work ability index. 2nd ed. Helsinki: Finnish Institute of Occuptional Health; 1998.

19. Ahlstrom L, Grimby-Ekman A, Hagberg M, Dellve L. The work ability index and single-item question: associations with sick leave, symptoms, and health-a prospective study of women on long-term sick leave. Scand J Work Environ Health. 2010;36(5):404-12.

20. Sell L. Predicting long-term sickness absence and early retirement pension from self-reported work ability. Int Arch Occup Environ Health. 2009;82(9):1133-8.

21. Nygård C-H, Arola H, Siukola A, Savinainen M, Luukkaala T, Taskinen H, Virtanen P. Perceived work ability and certified sickness absence among workers in a food industry. Int Congr Ser. 2005;1280:296-300.

22. Konstantinidis A, Martiny K, Bech P, Kasper S. A comparison of the major depression inventory (MDI) and the Beck depression inventory (BDI) in severely depressed patients. Int J Psychiatry Clin Pract. 2011;15(1):56-61.

23. Youden WJ. Index for rating diagnostic tests. Cancer. 1950;3(1):32-5.

24. Ruopp MD, Perkins NJ, Whitcomb BW, Schisterman EF. Youden index and optimal cut-point estimated from observations affected by a lower limit of detection. Biometrical J Biom Z. 2008;50(3):419-30.

25. Xiong C, DW MK Jr, Miller JP, Morris JC. Combining correlated diagnostic tests: application to neuropathologic diagnosis of Alzheimer's disease. Med Decis Making. 2004;24(6):659-69. 